\title{
The solar type protostar IRAS16293-2422: new constraints on the physical structure
}

\author{
N. Crimier ${ }^{1}$, C. Ceccarelli ${ }^{1}$, S. Maret ${ }^{1}$, S. Bottinelli ${ }^{2}$, E. Caux ${ }^{1}$, C. Kahane ${ }^{1}$, D. C. Lis $^{3}$, and J. Olofsson ${ }^{1}$ \\ 1 Laboratoire d'Astrophysique, Observatoire de Grenoble, 38041 Grenoble, France \\ e-mail: [Nicolas.Crimier;Cecilia.Ceccarelli; Sebastien.Maret;Claudine.Kahane]@obs.ujf-grenoble.fr \\ 2 CESR-UPS, Centre National de la Recherche Scientifique, Toulouse, France \\ e-mail: Sandrine. Bottinelli@cesr.fr \\ 3 California Institute of Technology, MC 301-17, Pasadena, CA 91125, USA \\ e-mail: dcl@caltech.edu
}

Received 12 August 2009 / Accepted 23 March 2010

\section{ABSTRACT}

\begin{abstract}
Context. The low mass protostar IRAS16293-2422 is a prototype Class 0 source in studies of the chemical structure during the initial phases of life of solar type stars.

Aims. To derive the chemical structure accurately, a precise determination of the source physical structure is required. The goal of this study is to derive the structure of IRAS16293-2422.

Methods. We reanalyzed all available continuum data (single dish and interferometric, from millimeter to MIR) to derive accurate density and dust temperature profiles. Using ISO observations of water, we also reconstructed the gas temperature profile.

Results. Our analysis shows that the envelope surrounding IRAS16293-2422 is described well by the Shu "inside-out" collapsing envelope model or a single power-law density profile with index equal to 1.8. In contrast to some previous studies, our analysis does not show evidence of a large ( $\geq 800$ AU in diameter) cavity.

Conclusions. Although IRAS16293-2422 is a multiple system composed of two or three objects, our reconstruction will be useful in deriving the chemical structure of the large cold envelope surrounding these objects and the warm component, treated here as a single source, from single-dish observations of molecular emission.
\end{abstract}

Key words. stars: formation - ISM: molecules - ISM: abundances

\section{Introduction}

Understanding how our Sun and the Solar System formed is arguably one of the major goals of modern astrophysics. Many different approaches contribute to our understanding of the past history of the Solar System. Analyzing the relics of the ancient eons, comets, and meteorites, is one. Studying present-day objects similar to the progenitor of the Sun is another. Here we pursue the latter approach and analyze in detail the case of one of the most well-studied solar-type protostars, IRAS16293-2422 (hereinafter IRAS16293). IRAS16293 is a Class 0 protostar in the $\rho$ Ophiuchus complex at $120 \mathrm{pc}$ from the Sun (Loinard et al. 2008) that has played a similar role as a prototype for solar-type protostars in astrochemical studies as Orion KL for high-mass protostars. This is because of its proximity and the strength of the lines of molecular emission (e.g., Walker et al. 1986; Mundy et al. 1992; Blake et al. 1994; van Dishoeck et al. 1995, to mention just a few representative works from the previous decades). It was in this source that the phenomenon of the "superdeuteration" "was first discovered, by detecting a surprisingly abundant amount of multiply deuterated molecules: formaldehyde, hydrogen sulfide, methanol, and water (Ceccarelli et al. 1998; Vastel et al. 2003; Parise et al. 2003; Butner et al. 2007).

\footnotetext{
1 The super-deuteration refers to the exceptionally high abundance ratio of D-bearing molecules with respect to their H-bearing isotopologues found in low mass protostars, with observed $\mathrm{D}$-molecule/Hmolecule ratios reaching the unity (see e.g., the review in Ceccarelli et al. 2007).
}

The first hot corino (Bottinelli et al. 2004; Ceccarelli et al. 2007) was also discovered in this source, with the detection of several abundant complex organic molecules in the region where the dust grain mantles sublimate (Ceccarelli et al. 2000b; Cazaux et al. 2003; Bottinelli et al. 2004).

Unsurprisingly, IRAS16293 has therefore been the target of several studies to reconstruct its physical structure, namely its dust and gas density and temperature profiles (Ceccarelli et al. 2000a; Schöier et al. 2002, 2004; Jørgensen et al. 2005), the mandatory first step to correctly evaluate the abundance of molecular species across the envelope. Ceccarelli et al. (2000a) used water and oxygen lines observations obtained with the Infrared Space Observatory (ISO) to derive the gas and dust density and temperature profile. In contrast, Schöier et al. (2002) used the dust continuum observations to derive the structure of the envelope. Moreover, Ceccarelli et al. assumed the semianalytical solution by Shu and coworkers (Shu 1977; Adams \& Shu 1986) to fit the observational data, while Schöier et al. (2002) assumed a single power law for the density distribution, and a posteriori verified that the Shu's solution also reproduced the observational data. The two methods lead to similar general conclusions: a) the envelope of IRAS16293 is centrally peaked and has a density distribution in overall agreement with the inside-out collapse picture (Shu 1977); b) there is a region, about $300 \mathrm{AU}$ in diameter, where the dust mantles sublimate (giving rise to the phenomenon of the hot corino, mentioned above). However, the two different methods, unsurprisingly, also lead to some notable differences. For example, the gas density differs by 
about a factor 3 in the region where the ice sublimation is predicted to occur, leading to differences in the derived abundances of several molecular species.

Subsequent studies have built on the early ones to improve the derivation of the physical structure of IRAS16293. First, the study by Schöier et al. (2004), based on interferometric OVRO observations, concluded that the envelope has a large central cavity, about $800 \mathrm{AU}$ in diameter. Using new Spitzer data, Jørgensen et al. (2005), then concluded that the envelope has an even larger central cavity, about 1200 AU in diameter. This large central cavity has a great impact on the whole interpretation of the hot corino of IRAS16293, because it predicts the absence of the mantle sublimation region. If the predicted cavity is real, the observed complex organic molecules must have an origin other than grain mantle sublimation from thermally heated dust. In addition to raising an important point in itself, Schöier et al. and Jørgensen et al. illustrate the paramount importance of correctly understanding the physical structure of the source in order to assess the chemical structure and all that follows.

In the present work, we therefore re-analyzed the available data on IRAS16293 from scratch, considering, in addition, the distance to this source estimated by Loinard et al. (2008) (120 pc instead of $160 \mathrm{pc}$, as assumed in Ceccarelli et al. 2000a, Schöier et al. 2002, 2004, and Jørgensen et al. 2005). This new analysis is necessary and timely because of two important observational projects having IRAS16293 as a target: a) the unbiased spectral survey in the $3,2,1$, and $0.8 \mathrm{~mm}$ bands just obtained at the IRAM and JCMT telescopes ("The IRAS16293-2422 Millimeter And Sub-millimeter Spectral Survey"2; Caux et al. 2010, in prep.), and b) the unbiased spectral survey between 500 and $2000 \mathrm{GHz}$ that will shortly be performed with the heterodyne instrument HIFI aboard the Herschel Space Observatory (HSO) launched in May 2009 (the Herschel Guaranteed Time Key Program CHESS - Chemical Herschel Surveys of Star Forming Regions ${ }^{3}$ ). The two projects, involving large international teams, will provide an accurate census of the molecular inventory of IRAS16293, the largest ever obtained for a solar type protostar. To convert the observations into an accurate chemical composition across the IRAS16293 envelope, the dust and gas density and temperature profiles have first to be determined accurately. Deriving these profiles is the goal of the present article.

We conclude this section by addressing the problem of the binarity of IRAS16293 and how it agrees with the analysis we present here. As soon as interferometric observations became available it was realized that IRAS16293 is indeed a protobinary system (Wootten 1989; Mundy et al. 1992), composed of two sources: A (the south source) and B (the north source) separated by $\sim 4^{\prime \prime}$, i.e., $\sim 500 \mathrm{AU}$ at $120 \mathrm{pc}$. While source B is the brightest in the continuum, source $\mathrm{A}$ is often, but not always, the brightest in the molecular emission (e.g., Chandler et al. 2005). The most recent observations show that IRAS16293 is indeed a triple system, the source A being composed of two objects, A1 and A2, of 0.5 and $1.5 M_{\odot}$, respectively (Loinard et al. 2009). While it is clear that the multiple nature of IRAS16293 cannot be neglected in general, the two projects mentioned above involve observations with single-dish telescopes, so that much of the structure on small scal es is smeared out in these observations. The molecular line emission will be dominated by the cold envelope, which fills the telescope beam, and by any warm component at the interior of the envelope. The major goal here

\footnotetext{
2 http://www-laog.obs.ujf-grenoble.fr/heberges/ timasss/

3 http://www-laog.obs.ujf-grenoble.fr/heberges/chess/
}

is to provide a reliable estimate of the envelope temperature and density profiles of both the gas and dust components, up to the scales where the approximation of a spherical symmetry is valid. We later discuss the size of these scales, based on the available observations.

The article is organized as follows. Section 2 discusses the derivation of the dust density and temperature distribution, based on the analysis of all available continuum data. Section 3 describes the derivation of the gas temperature profile, and the constraint of the abundance of a major gas coolant with the help of ISO. Finally, Sect. 4 discusses and summarizes the results of the presented study.

\section{Dust temperature and density profiles}

\subsection{The data set}

The present analysis is based on the continuum emission from the envelope that forms/surrounds the protostar IRAS16293. Three types of observations are considered: maps of the emission, a spectral energy distribution (SED), and interferometric observations at 1 and $3 \mathrm{~mm}$. All data used had been retrieved from archives, except the map at $350 \mu \mathrm{m}$ and the 1 and $3 \mathrm{~mm}$ interferometric data that we obtained by means of dedicated observations. Below we briefly describe the data used:

\section{i) Continuum emission profiles}

We used the maps of the dust continuum emission at $350 \mu \mathrm{m}$ (obtained at the Caltech Submillimeter Observatory; CSO), and 450 and $850 \mu \mathrm{m}$ (obtained at the James Clerk Maxwell Telescope; JCMT). The 450 and $850 \mu \mathrm{m}$ maps had been retrieved from the JCMT archive (website). The beam sizes are $7.5^{\prime \prime}$ and $14.8^{\prime \prime}$ at 450 and $850 \mu \mathrm{m}$, respectively. Based on the many previous JCMT published observations, the calibration uncertainty and noise levels are $\lesssim 10 \%$ and $0.04 \mathrm{Jy} \mathrm{beam}^{-1}$ at $850 \mu \mathrm{m}$ and $\lesssim 30 \%$ and $0.3 \mathrm{Jy} \mathrm{beam}^{-1}$ at $450 \mu \mathrm{m}$, respectively.

Observations of the $350 \mu \mathrm{m}$ continuum emission toward IRAS 16293 reported here were carried out in 2003 February using the SHARC II facility bolometer camera of the Caltech Submillimeter Observatory (CSO) on Mauna Kea in Hawaii (Dowell et al. 2003). SHARC II is a $12 \times 32$ pixel filled array with a field of view of $2.6 \times 1.0^{\prime}$. The data were taken during excellent submillimeter weather conditions (a $225 \mathrm{GHz}$ zenith opacity of 0.04 , corresponding to less than $1 \mathrm{~mm}$ of precipitable water). The observations were carried out using the "box-scan" scanning mode ${ }^{4}$. Five 10 min scans were reduced together using the CRUSH software package (Kovács 2008 ) to produce the final calibrated image. Telescope pointing was checked by observing Juno, Vesta, or Mars, immediately before or after the science observations and the measured offsets were applied during data reduction. The data were taken before the CSO Dish Surface Optimization system (DSOS) become operational. The shape of the telescope beam was determined from the pointing images of Vesta and Mars. It contains a diffraction-limited main beam with a $F W H M$ diameter of $9^{\prime \prime}$ and an error beam with a FWHM diameter of $22^{\prime \prime}$, with relative peak intensities of 0.8 and 0.2 , respectively. This size of the error beam is consistent with earlier $350 \mu \mathrm{m}$ measurements using the SHARC I camera (Hunter 1997).

We obtained continuum emission profiles as a function of the distance from the center of the envelope, by averaging

\footnotetext{
4 see http: //WWW . submm . caltech. edu/sharc/
} 

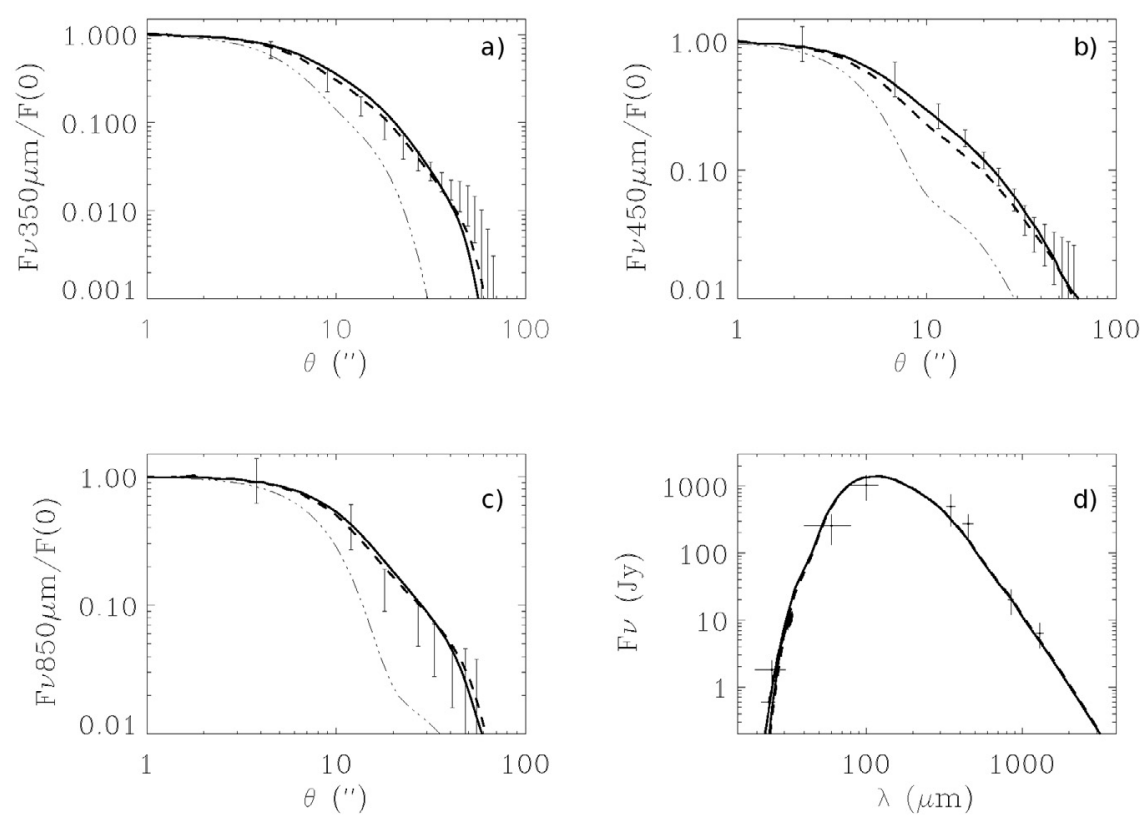

Fig. 1. Continuum emission profiles at $850 \mu \mathrm{m}$ (left bottom panel), $450 \mu \mathrm{m}$ (right upper panel) and $350 \mu \mathrm{m}$ (left upper panel), plus the SED (right bottom panel). The curves show the emission predicted by two models with different density profiles (see text for more details): the solid thick line is the best-fit of the maps and SED data obtained by a density profile with a 2-indexes power law with $\alpha_{\text {out }}=2$ and $\alpha_{\text {in }}=1.5$ in the outer and inner envelope respectively (Shu-like density distribution); the dashed line is the best-fit obtained by a single power-law index, $\alpha=1.8$. The dotted-dashed lines show the telescope beam profiles adopted.

Table 1. The spectral energy distribution of IRAS 16293-2422.

\begin{tabular}{lllll}
\hline \hline $\begin{array}{l}\lambda \\
(\mu \mathrm{m})\end{array}$ & $\begin{array}{l}\mathrm{F}_{v}{ }^{a} \\
(\mathrm{Jy})\end{array}$ & $\begin{array}{l}\Delta \mathrm{F}_{v}{ }^{b} \\
(\mathrm{Jy})\end{array}$ & $\begin{array}{l}\theta_{\mathrm{mb}^{c}} \\
\left({ }^{\prime \prime}\right)\end{array}$ & Ref. \\
\hline 23.7 & 0.6 & 0.1 & 6.0 & 2 \\
25 & 1.8 & 0.7 & 80.0 & 3 \\
60 & 255. & 122. & 160.0 & 3 \\
100 & 1032. & 412. & 237.0 & 3 \\
350 & 500. & 250. & 9.0 & 1 \\
450 & 270. & 108. & 7.8 & 1 \\
850 & 20.2 & 8. & 14.5 & 1 \\
1300 & 6.4 & 2.6 & 22.0 & 4 \\
\hline
\end{tabular}

Notes. ${ }^{(a)}$ Integrated flux in Jy . ${ }^{(b)}$ Uncertainties in Jy considering the calibration uncertainty, the noise levels, and the uncertainty in the source size. ${ }^{(c)}$ Main beam of the instrument in arcsec.

References. (1) This paper; (2) Spitzer catalog; (3) IRAS Point Source Catalog v2.1; (4) Saraceno et al. (1996).

the map emission over annuli at the same distance. In each case, we adopted a radial sampling corresponding to the half of the resolution of the instrument. The uncertainties in the continuum emission profiles were evaluated taking into account the calibration uncertainty, noise levels, and the nonsphericity of the source. The resulting profiles, normalized to the peak emission, are shown in Fig. 1. We note that the 450 and $850 \mu \mathrm{m}$ profiles are identical to those reported by Schöier et al. (2002).

ii) Spectral energy distribution

Table 1 reports the SED obtained by considering all the data available in the literature (plus the $350 \mu \mathrm{m}$ point obtained by us; see above). The millimeter and submillimeter data points were obtained by integrating the maps over the entire envelope. The integrated flux at $1.3 \mathrm{~mm}$ was that quoted in Saraceno et al. (1996). We retrieved the IRAS fluxes from the IRAS Point Source Catalog v2.1 ${ }^{5}$.

\footnotetext{
${ }^{5}$ http://irsa.ipac.caltech.edu/cgi-bin/Gator/nph-dd? catalog=iraspsc
}

IRAS 16293 was observed with the InfraRed Spectrograph (IRS) installed aboard the Spitzer Space Telescope as part of the "From Molecular Cores to Planet Forming DIsks" (Evans et al. 2003; Evans \& c2d Team 2005) Legacy Program (AOR: 11826944, PI: Neal Evans). We used the observations obtained with the Long-High (LH) module (20-37 $\mu \mathrm{m}, R=$ 600 ) on 2004 July 29, in staring mode. The data reduction was performed using the c2d pipeline S15.3.0 (Lahuis et al. 2006) with the pre-reduced (BCD) data. Since the MIPS map at $24 \mu \mathrm{m}$ shows that the IRAS16293 emitting region $(\sim 30-$ $\left.40^{\prime \prime}\right)$ is larger than the LH module field of view $\left(11.1^{\prime \prime} \times\right.$ $22.3^{\prime \prime}$ ), we adopted the full aperture extraction method in the pipeline. In addition, we corrected the derived flux level for the missing flux by comparing the IRS spectrum integrated on the MIPS bandwith and the integrated MIPS flux. We note that this method assumes that the source emission has a similar distribution across the whole IRS wavelength interval, $\sim 21-37 \mu \mathrm{m}$. The correction factor derived by this method is $22 \%$ in the flux.

iii) Interferometric continuum data

Observations of the 3 and $1.3 \mathrm{~mm}$ continuum were obtained with the IRAM Plateau de Bure Interferometer (PdBI) in 2004 and are described in detail in Bottinelli et al. (2004). They were obtained using the $\mathrm{B}$ and $\mathrm{C}$ configurations of the PdBI array, resulting in a spatial resolution of about $0.8^{\prime \prime}$.

\subsection{Adopted model}

We obtained the best-fit to the continuum data, described in the previous section, by using the 1D radiative transfer code DUSTY (Ivezic \& Elitzur 1997), which has been extensively used in similar works (including Jørgensen et al. 2002, 2005 Schöier et al. 2002, 2004). Briefly, providing as input the temperature of the central object and a dust density profile, DUSTY selfconsistently computes the dust temperature profile and the dust emission. A comparison between the computed 350, 450, and $850 \mu \mathrm{m}$ brightness profiles (namely the brightness versus the distance from the center of the envelope) and SED derived for the observations (described in the previous section) allows us to 
constrain the density profile and, consequently, the temperature profile of the envelope. To be able to compare it with the observations, the theoretical emission is convolved with the beam pattern of the telescope. Following the recommendations for the relevant telescope, the beam is assumed to be a combination of Gaussian curves: at $850 \mu \mathrm{m}$, we use HPBWs of $14.5^{\prime \prime}, 60^{\prime \prime}$, and $120^{\prime \prime}$, with amplitudes of $0.976,0.022$, and 0.002 respectively; at $450 \mu \mathrm{m}$, the HPBWs are $8^{\prime \prime}, 30^{\prime \prime}$, and $120^{\prime \prime}$ with amplitude ratios of $0.934,0.06$, and 0.006 , respectively (Sandell \& Weintraub 2001 ); at $350 \mu \mathrm{m}$, we use HPBWs of $9^{\prime \prime}$ and $22^{\prime \prime}$, with amplitude ratios of $0.8,0.2$, respectively (Sect. 2.1).

In this work, we consider two cases for the density distribution. In the first, we assume a broken power-law density profile as in the Shu (1977) solution

$$
\begin{array}{ll}
n(r)=n\left(r_{0}\right)\left(\frac{r_{0}}{r}\right)^{1.5} & r \leq r_{\mathrm{inf}} \\
n(r)=n\left(r_{0}\right)\left(\frac{r_{0}}{r}\right)^{2} & r \geq r_{\mathrm{inf}}
\end{array}
$$

where $r_{\text {inf }}$ is the radius of the collapsing envelope (at larger radii the envelope is static). In the density profile, it represents the radius at which the change of index occurs and it is a free parameter. We note that the complete Shu (1977) solution also contains a transition part at the interface of the collapsing and static regions, just inside $r_{\text {inf }}$. In this region, the slope gradually changes from $r^{-1}$ to the limiting value of $r^{-1.5}$. A posteriori, the effective part of the envelope in $r^{-1}$ is relatively small and located in the inner region $\left(\lesssim 1500 \mathrm{AU}\right.$, equivalent to $\left.\sim 10^{\prime \prime}\right)$. Since the continuum observations, with resolutions of $\sim 8-18^{\prime \prime}$, are not sensitive enough, we adopted the simplified structure described by Eqs. (1), (2). In the second case, we considered a single powerlaw density profile, where the index $\alpha$ is a free parameter

$n(r)=n\left(r_{0}\right)\left(\frac{r_{0}}{r}\right)^{\alpha}$.

In both cases, $n\left(r_{0}\right)$ is the density at $r_{0}$, and the envelope starts at a radius $r_{\text {in }}$ and extends to $r_{\text {out }}$. In total, both models have four free parameters determined by the best-fit solution for the observational data: $r_{\text {inf }}$ or $\alpha, n\left(r_{0}\right), r_{\text {out }}$, and $r_{\text {in }}$. Finally, DUSTY requires the temperature of the central source, $T_{*}$, here assumed to be $5000 \mathrm{~K}$. We note that we verified that the choice of this parameter does not influence the results, as already noticed by other authors (e.g., Jørgensen et al. 2002). In practice, the DUSTY input parameters are the infall radius $r_{\text {inf }}$ or the power-law index $\alpha$, the optical thickness at $100 \mu \mathrm{m}, \tau_{100}$, the ratio of the inner to outer radius, $Y\left(=r_{\text {out }} / r_{\text {in }}\right)$, and the temperature at the inner radius $T_{\text {in }}{ }^{6}$. The optical thickness is, in turn, proportional to $n\left(r_{0}\right)$ and $r_{\text {out }}$. In both models, we obtain a lower limit to $T_{i n}$ of $300 \mathrm{~K}$, any higher value giving similar results.

In addition to the above parameters, the opacity of the dust as a function of wavelength is a parameter of DUSTY. Following numerous previous studies (Van Der Tak et al. 1999; Evans et al. 2001; Shirley et al. 2002; Young et al. 2003; Schöier et al. 2002), we adopted the dust opacity calculated by Ossenkopf \& Henning (1994), namely their OH5 dust model, which refers to grains coated by ice. Again, the basic result, though, does not substantially depend on the choice of the dust opacity model.

As explained in Ivezic \& Elitzur (1997), DUSTY gives scalefree results, so that the source bolometric luminosity $L_{\mathrm{bol}}$ and the

\footnotetext{
6 The temperature at the inner radius $T_{\text {in }}$ defines the radius at which the integration starts. The DUSTY codes requires the temperature rather than the radius because it is based on a scale-free algorithm.
}

Table 2. Range and step of the DUSTY input parameters.

\begin{tabular}{lll}
\hline \hline Parameter & Range & Step \\
\hline$\alpha$ & $0.2-2.5$ & 0.1 \\
$Y^{a}$ & $50-2000$ & 10 \\
$r_{\text {inf }}$ & $5-200$ & 2 \\
$\tau_{100}$ & $0.1-10$. & 0.1 \\
$T_{\text {in }}$ & $300 \mathrm{~K}$ & Fixed \\
$T_{*}$ & $5000 \mathrm{~K}$ & Fixed \\
\hline
\end{tabular}

Notes. ${ }^{(a)} Y=r_{\text {out }} / r_{\text {in }}$.

distance are required to compare the DUSTY output with actual observations. We assumed the lastest estimate of the distance to the $\rho$ Ophiuchus cloud, namely 120 pc (see Introduction), and we derived the bolometric luminosity by integrating the observed emission over the full spectrum, optimizing the resulting $\chi^{2}$ (see below).

We computed grids of models for both cases described above. The summary of the covered parameter space is reported in Table 2. The best-fit model was found by minimizing the $\chi^{2}$ with an iterated two-step procedure (see also Crimier et al. 2009). First, we use the observed brightness profiles at 350, 450 and $850 \mu \mathrm{m}$ to constrain $Y$ and $\alpha$ (or $r_{\text {inf }}$ in the case of the Shulike model), assuming a value for $\tau_{100}$. Second, we constrain the optical thickness $\tau_{100}$ by comparing the computed and observed SED, assuming the $\alpha$ (or $r_{\text {inf }}$ ) and $Y$ of the previous step. The new $\tau_{100}$ is used for a new iteration and so on. In practice, the iteration converges in two steps because the normalized brightness profiles depend very weakly on $\tau_{100}$, while they very much depend on the assumed size of the envelope and on the slope of the density profile (see the discussion in Jørgensen et al. 2002; Crimier et al. 2009). In contrast, the dust optical thickness depends mostly on the absolute density of the envelope. We note that we also constrain the bolometric luminosity based on the best-fit model of the $\mathrm{SED}^{7}$.

\subsection{Results}

\section{a) Brightness profiles and SED analysis}

Table 3 lists the best-fit model parameters for the two models described in the previous section as well as the parameters obtained by Schöier et al. (2002) for comparison. Figure 1 compares the solutions to the observed data (maps and SED).

The results of this part of the analysis are:

- The bolometric luminosity, obtained by minimizing the $\chi^{2}$, is $22 L_{\odot}$ rather then $14 L_{\odot}$, the value obtained by simply scaling the bolometric luminosity adopted by Ceccarelli et al. (2000b) and Schöier et al. (2002) to a distance of $120 \mathrm{pc}$ instead of $160 \mathrm{pc}$.

- The single power-law and Shu-like density distributions can both reproduce the observations, the maps, and the SED data, including the IR part of the spectrum (Fig. 1). They provide similar best-fit $\chi^{2}$ values, although the Shu-like distribution reproduces more accurately the $450 \mu \mathrm{m}$ profile. Also the derived physical parameters are substantially similar for the two models.

- The observed data, including the Spitzer data between 20 and $40 \mu \mathrm{m}$, do not imply that a large cavity must be present

7 We note that the dust optical thickness $\tau_{100}$ affects the shape of the SED, so in general it enters into the determination of the bolometric luminosity. 
Table 3. Summary of the dust radiative transfer analysis.

\begin{tabular}{|c|c|c|c|}
\hline Quantity & Shu-like model & Power-law model & Schöier et al. (2002) \\
\hline \multicolumn{4}{|c|}{ Best - fit parameters } \\
\hline$L_{*}\left(L_{\odot}\right)$ & 22 & 22 & 27 \\
\hline$D(\mathrm{pc})$ & 120 & 120 & 160 \\
\hline$\tau_{100}$ & 2.0 & 3.0 & 4.5 \\
\hline$r_{\text {inf }}(\mathrm{AU})$ & 1280 & & \\
\hline$\alpha$ & & 1.8 & 1.7 \\
\hline$Y$ & 280 & 260 & 250 \\
\hline \multicolumn{4}{|c|}{ Physical quantities } \\
\hline$r_{\text {in }}(\mathrm{AU})$ & 22 & 27 & 32 \\
\hline$r_{\text {out }}(\mathrm{AU})$ & 6100 & 6900 & 8000 \\
\hline $\mathrm{r}\left(T_{\text {dust }}=100 \mathrm{~K}\right)(\mathrm{AU})$ & 76 & 85 & \\
\hline $\mathrm{n}\left(T_{\text {dust }}=100 \mathrm{~K}\right)\left(\mathrm{cm}^{-3}\right)$ & $2 \times 10^{8}$ & $3 \times 10^{8}$ & \\
\hline$M_{\text {env }}\left(M_{\odot}\right)$ & 1.9 & 2.1 & 5.4 \\
\hline
\end{tabular}

Notes. The upper half of the table lists the best-fit model parameters, the lower half reports some relevant physical quantities. The last column reports the results of the Schöier et al. (2002) analysis for comparison.

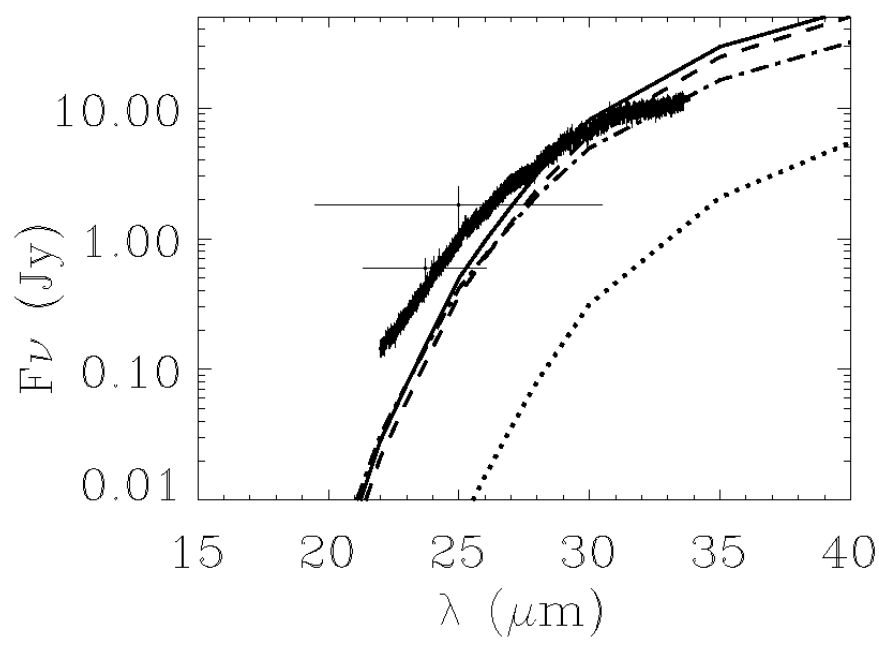

Fig. 2. MIR Spitzer and IRAS fluxes as a function of the wavelength. The two crosses represent the integrated fluxes at 23.7 and $25 \mu \mathrm{m}$ obtained with MIPS aboard Spitzer and CPC aboard IRAS, respectively. The solid thick line is the IRS Spitzer spectrum observed between $\sim 21-$ $37 \mu \mathrm{m}$ (see text for details). The solid thin line is the best-fit model of the maps and SED data given by an envelope with a density profile with a broken power law with $\alpha_{\text {out }}=2$ and $\alpha_{\text {in }}=1.5$ in the outer and inner envelope, respectively (Shu-like density distribution). The dashed line is the best-fit solution given by the model of envelope with a single power-law index, $\alpha=1.8$. The dotted-dashed line and the dotted lines show the emission resulting from the models obtained by Jørgensen et al. (2005) and Schöier et al. (2002), respectively.

(Jørgensen et al. 2005). Our solution using higher luminosity (22 instead of $14 L_{\odot}$ ) also can reproduce the MIR Spitzer observations. Figure 2 compares the MIR Spitzer and IRAS observations with the emission predicted by our models and those of Jørgensen et al. (2005) and Schöier et al. (2002).

Figure 3 shows the derived dust temperature and density profiles. The single power-law index density distribution predicts a slightly warmer and denser region at radii smaller than about $100 \mathrm{AU}$, but the differences are relatively small (see also Table 3).

\section{b) Interferometric data analysis}

The previous analysis considers single dish data, which at best have a spatial resolution of $8^{\prime \prime}$, equivalent to a radius of
Table 4. Integrated flux and full width at half maximum $(F W H M)$ of the Gaussians used to model the emission of the sources A and B at 1.3 and $3 \mathrm{~mm}$.

\begin{tabular}{lllll}
\hline \hline & & $1.3 \mathrm{~mm}$ & & $3 \mathrm{~mm}$ \\
\hline Source & $\mathrm{F}_{v}(\mathrm{Jy})$ & $F W H M\left({ }^{\prime \prime}\right)$ & $F_{v}(\mathrm{Jy})$ & $F W H M\left({ }^{\prime \prime}\right)$ \\
\hline $\mathrm{A}$ & 0.49 & 1.18 & 0.16 & 1.62 \\
$\mathrm{~B}$ & 0.97 & 0.86 & 0.25 & 0.78 \\
\hline
\end{tabular}

$\sim 1000$ AU. To constrain the inner region in greater detail, we used 1 and $3 \mathrm{~mm}$ continuum interferometric observations obtained with the Plateau de Bure interferometer (PdBI) described in the previous section. To compare the model predictions with the observations, we produced synthetic maps for each model, to which we added the predicted emission from the envelope plus the emission of the two Gaussian-like sources A and B. The parameters used to represent the emission of the sources A and B were extracted from the interferometric maps at 1.3 and $3 \mathrm{~mm}$ and reported in Table 4. We then used the UV_FMODEL task in Gildas ${ }^{8}$ to compute visibility tables with the same $u v$ plane coverage as the actual observations.

Table 5 reports the $\chi_{\text {red }}^{2}$ values obtained using the observed and modeled visibility amplitudes at $1.3 \mathrm{~mm}$. The comparison is performed for each of the three models of the envelope, i.e., the single power-law density distribution, the Shu-like density distribution, and the central cavity of Schöier et al. (2004). We tested cases in which the envelope is centered on one of the two sources or on the mid-way point between the two sources. The $\chi_{\text {red }}^{2}$ values are computed over more than $10^{4}$ points. The $2 \mathrm{D}$ representation of the visibility amplitudes observed and modeled in $u v$ plan is difficult to read. Therefore to illustrate the comparison between the visibility amplitudes observed and modeled, we averaged the visibility amplitudes over the same $u v$ radius and plotted them in Fig. 4. We note that the error bars shown in Fig. 4 represent only the measurement errors. The standard deviations resulting from the radial average are meaningless in this case due to its non-sphericial geometry (caused by the sources A and B). Figure 4 attempts to illustrate the comparison between the observations and the models, our conclusions being based on the $\chi_{\text {red }}^{2}$ in Table 5. The computations were performed considering all the visibility amplitudes obtained in the $u v$ plane before the azimuthal average.

\footnotetext{
8 http://iram.fr/IRAMFR/GILDAS/doc/html/mis-html/ node13.html
} 
A\&A 519, A65 (2010)
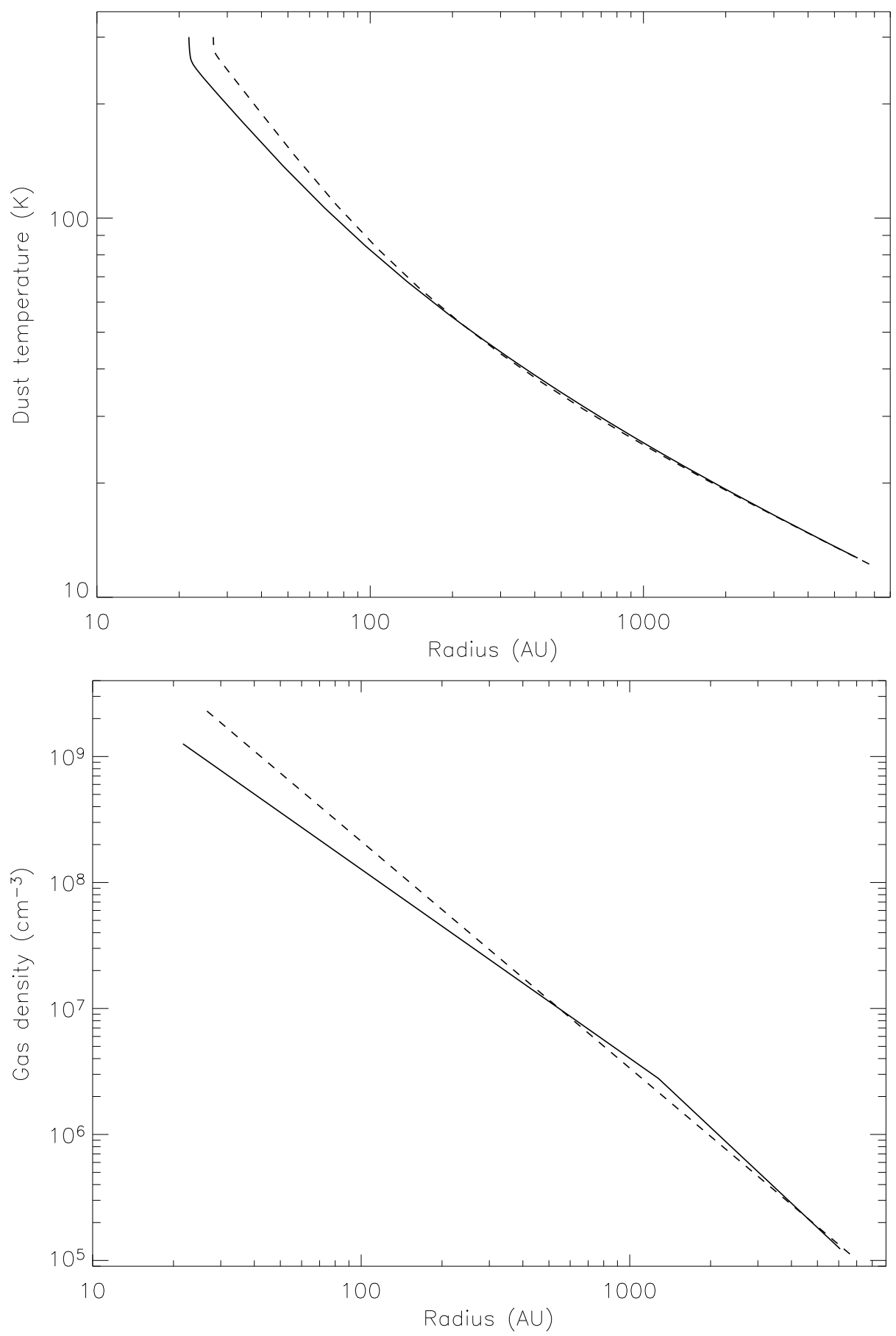

Fig. 3. Dust temperature (upper panel) and $\mathrm{H}_{2}$ density (lower panel) profiles from the bestfit obtained in the two cases Shu-like (solid line) and single power-law density distribution (dashed line).

Table 5. $\chi_{\text {red }}^{2}$ values obtained comparing the visibility amplitudes observed and modeled at $1.3 \mathrm{~mm}$.

\begin{tabular}{lccc}
\hline \hline$\lambda=1.3 \mathrm{~mm}$ & $\begin{array}{c}\text { Model centered } \\
\text { in between A and B }\end{array}$ & $\begin{array}{c}\text { Model centered } \\
\text { on B }\end{array}$ & $\begin{array}{c}\text { Model centered } \\
\text { on A }\end{array}$ \\
\hline Model & $\chi_{\text {red }}^{2}$ & $\chi_{\text {red }}^{2}$ & $\chi_{\text {red }}^{2}$ \\
\hline Single power-law & 18.5 & 10.2 & 8.5 \\
Shu-like & 13.3 & 10.3 & 8.3 \\
Central cavity & 11.0 & 11.3 & 9.9 \\
\hline
\end{tabular}

Notes. The number of degrees of freedom $v$ is 10230. The 1 st column reports the $\chi_{\text {red }}^{2}$ obtained using the synthetic maps with the envelope centered on the mid-way point between the sources A and B. The 2 nd and the 3 rd columns report the $\chi_{\text {red }}^{2}$ obtained using the synthetic maps with the envelope centered on the source B and on the source A, respectively. The 1st, the 2nd, and the 3rd lines report the $\chi_{\text {red }}^{2}$ obtained in all the cases discussed in the text, using the model of envelope with a single power-law density distribution, a Shu-like density distribution, and a central cavity of Schöier et al. (2004), respectively. 
The interferometric data are dominated by the two components of the IRAS16293 binary system. However, regarding the envelope contribution - which is relevant to the present work in the case where the envelope is centered on the mid-way point between the sources $\mathrm{A}$ and $\mathrm{B}$, the visibilities at $u v$ radii lower than about $80 \mathrm{k} \lambda$ are not reproduced well by the single powerlaw density profiles. Our solution with the Shu-like density profile and the solution with a cavity (suggested by Schöier et al. 2004) provides far closer agreement. However, when envelope is centered on either B or A, all three models give similar results (Fig. 4), and reproduce the observed visibilities well. Since with the data available in the literature there is no way to know whether the envelope is centered on one of the two sources (A or B) or just on the mid-way point between them, either of the two solutions (centered on a source or on the mid-way point between A and B) is equally plausible. In other words, we are affected by a degeneracy dominated by the parameters relative to the binarity of the source. The interferometric data can be reproduced without a cavity. We note that the $3 \mathrm{~mm}$ PdBI observations do not provide additional information. We emphasize that the above analysis of the interferometric data is directly applicable to the OVRO data that led Schöier et al. (2004) to suggest the presence of a cavity. The OVRO data probe visibility amplitudes lower than about $60 \mathrm{k} \lambda$, a range also probed by the PDBI data. Therefore, as shown above, they can be reproduced by assuming that the envelope is centered on either source A or B without the necessity of assuming a cavity.

\section{c) Summary of the continuum data analysis}

Both the single dish and interferometric continuum data can be reproduced by either of the two models we considered: a Shulike and single power-law density distribution. Furthermore, no cavity is required to explain the data, neither the Spitzer MIR data (Jørgensen et al. 2005) nor the interferometric data (Schöier et al. 2004).

Therefore, the Shu-like density profile (which has a physical interpretation), with no cavity, is adopted as our reference model for the study of the gas temperature and water line predictions.

\section{Gas temperature profile}

\subsection{Adopted method}

We computed the gas temperature profile using the CHT96 code described in Ceccarelli et al. (1996) (see also Ceccarelli et al. 2000a; Maret et al. 2002; Crimier et al. 2009). Briefly, the code computes the gas equilibrium temperature at each point of the envelope, by equating the heating and cooling terms at each point of the envelope. Following the method described in Ceccarelli et al. (1996), we considered heating from the gas compression (due to the collapse), dust-gas collisions, and photopumping of $\mathrm{H}_{2} \mathrm{O}$ and $\mathrm{CO}$ molecules by the IR photons emitted by the warm dust close to the center ${ }^{9}$. The cooling terms are the line emission from $\mathrm{H}_{2} \mathrm{O}, \mathrm{CO}$, and $\mathrm{O}$. The dust-gas collisions are a source of heating when the dust temperature is higher than the gas temperature, and otherwise a source of cooling. To compute the cooling from the lines, we used the code described in Ceccarelli et al. (1996, 2003) and Parise et al. (2005). The same code has been used in several past studies, whose results have been substantially confirmed by other groups (e.g., the analysis

\footnotetext{
9 Cosmic ray ionization is a minor heating term in the protostellar envelopes.
}

on IRAS16293-2422 by Schöier et al. 2002). Briefly, the code is based on the escape probability formalism in the presence of warm dust (see Takahashi et al. 1983), where the escape probability $\beta$ is computed at each point by integrating the line and dust absorption over the solid angle $\Omega$ as follows:

$\beta=\frac{k_{\mathrm{d}}}{k_{\mathrm{L}}+k_{\mathrm{d}}}+\frac{k_{\mathrm{L}}}{\left(k_{\mathrm{L}}+k_{\mathrm{d}}\right)^{2}} \int \mathrm{d} \mu \frac{1-\exp \left[-\left(k_{\mathrm{L}}+k_{\mathrm{d}}\right) \Delta L_{\mathrm{th}}\right]}{\Delta L_{\mathrm{th}}}$

where $k_{\mathrm{L}}$ and $k_{\mathrm{d}}$ are the line and dust absorption coefficients, respectively, and $\Delta L_{\mathrm{th}}$ is the line trapping region, given by the expressions

$\Delta L_{\mathrm{th}}=2 \Delta v_{\mathrm{th}}\left(\frac{v}{r}\left|1-\frac{3}{2} \mu^{2}\right|\right)^{-1}$

in the infalling region of the envelope (where $\operatorname{arcos} \mu$ is the angle with the radial outward direction) and in the static region (where $R_{\text {env }}$ is the envelope radius)

$\Delta L_{\mathrm{th}}=r\left(1-\frac{r}{R_{\mathrm{env}}}\right)$

In addition, $\mathrm{H}_{2} \mathrm{O}$ and $\mathrm{CO}$ molecules can be pumped by absorption of the NIR photons emitted by the innermost warm dust. Since the densities and temperatures of the regions of the envelope targeted by this study are not enough to populate the levels in the vibrational states, the effect of the NIR photons is an extra heating of the gas, as described in Ceccarelli et al. (1996). We note that the code takes into account the dust with temperatures up to $1500 \mathrm{~K}$.

The code has a number of parameters that influence the gas equilibrium temperature. First, the dust temperature, assumed to be the output of the previous step analysis (Sect. 2). Second, since the infall heating depends on the velocity gradient across the envelope and the cooling depends on the gas lines (which can be and sometimes are optically thick), the velocity field across the envelope is also a parameter of the code. Here we assumed that the infall velocity field corresponds to the free fall velocity field with a $2 M_{\odot}$ source at the center of the envelope (Loinard et al. 2009). Third, we adopted the "standard" cosmic ray ionization rate, namely $3 \times 10^{-17} \mathrm{~s}^{-1}$, although in practcie this parameter is unimportant. Finally, given the contributions of the $\mathrm{H}_{2} \mathrm{O}$, CO, and OI to the gas cooling, their respective abundances are important parameters of the model. Previous theoretical studies have shown that the $\mathrm{O}$ abundance is constant across the envelope, except in the very inner regions, where the gas temperature exceeds about $250 \mathrm{~K}$, and endothermic reactions that form $\mathrm{OH}$ and $\mathrm{H}_{2} \mathrm{O}$ become very efficient (Ceccarelli et al. 1996; Doty \& Neufeld 1997; Doty et al. 2004). Unfortunately, this parameter is very poorly constrained by observations (because of the difficulty in observing the $\mathrm{O}$ fine structure lines and their being easily excited in the foreground molecular cloud and its associated PDR (see e.g., Liseau et al. 1999; Caux et al. 1999). Here we assumed that the atomic $O$ abundance is equal to $1 \times 10^{-5}$ and verified a posteriori that the $\mathrm{O}$ fine structure line emission is consistent with the ISO observations. We note, however, that $\mathrm{O}$ is never the dominant coolant except, perhaps, in a very small region of the envelope (Ceccarelli et al. 2000a; Maret et al. 2002). In contrast, CO, is the main coolant in the outer envelope, but, since the cooling lines are heavily optically thick, its abundance does not play an important role (in the regime where it is higher than about $1 \times 10^{-6}$ with respect to $\mathrm{H}_{2}$ ). We, 


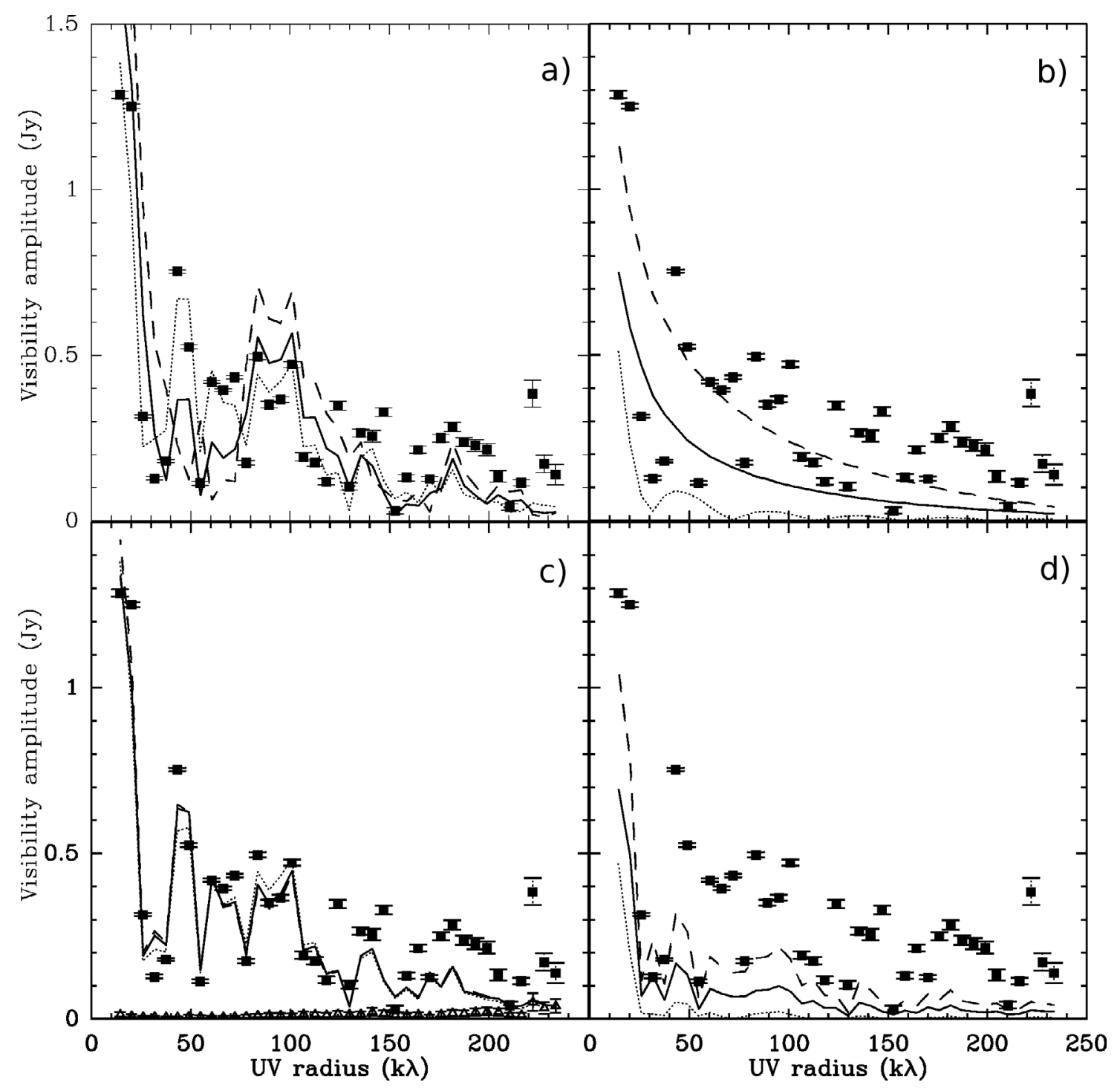

Fig. 4. Visibility amplitudes as a function of the $u v$ radius of the $1.3 \mathrm{~mm}$ PdBI observations (squares) against the predictions obtained with the best-fit models of a Shu-like (solid lines) and single power-law (dashed lines) density distribution. The model of Schöier et al. (2004), with the central cavity, is shown for a comparison (dotted lines). Panels a) and b) show the case where the envelope is centered on the mid-way point between the sources A and B. Panels c) and d) show the case where the envelope is centered on the source B. Panels a) and c) show the visibility amplitudes modeled with the synthetic maps containing the contribution of the envelope and the sources A and B. Panels b) and d) show the visibility amplitudes modeled with the synthetic maps containing the contribution of the envelope only. Finally, the zero-signal expectation values are represented by the empty triangles on the c) panel.

therefore, assumed a canonical $1 \times 10^{-4} \mathrm{CO}$ abundance across the envelope $\mathrm{e}^{10}$.

Finally, water is an important coolant in the inner region, where the grain mantles sublimate, injecting into the gas phase large quantities of water molecules. Based on previous studies, we approximated the $\mathrm{H}_{2} \mathrm{O}$ abundance with a step function: the abundance is $X_{\text {in }}$ in the region where the dust temperature exceeds $100 \mathrm{~K}$, and $X_{\text {out }}$ elsewhere. Both $X_{\text {in }}$ and $X_{\text {out }}$ are found by comparing the theoretical predictions with the ISO observations (see Sect. 3.2). We note that, to solve the water level population statistical equilibrium equations, we used the collisional coefficients between $\mathrm{H}_{2} \mathrm{O}$ and $\mathrm{H}_{2}$ computed by Faure et al. (2007). We assumed that the ortho-to-para $\mathrm{H}_{2}$ ratio is at the local

\footnotetext{
10 We note that it possible that a region where $\mathrm{CO}$ abundance is lower, because of the freezing onto the dust grains, exists. However, the gas cooling is relatively insensitive to this lower abundance for the reasons explained in the text.
}

thermal equilibrium (LTE) in each part of the envelope. Finally, we assumed a $\mathrm{H}_{2} \mathrm{O}$ ortho-to-para ratio equal to 3 .

\subsection{Results}

To constrain the water abundance, a necessary step in predicting the gas temperature profile, we used the ISO observations of the water lines towards IRAS16293 (Ceccarelli et al. 1998, 2000a) and compared them with the model predictions obtained for different $\mathrm{H}_{2} \mathrm{O}$ abundance profiles. We computed a grid of models with $X_{\text {in }}$ and $X_{\text {out }}$ varying between $1 \times 10^{-8}$ and $1 \times 10^{-5}$. Figure 5 shows the resulting $\chi^{2}$ as a function of the inner and outer $\mathrm{H}_{2} \mathrm{O}$ abundance, in the case of the Shu-like structure of Table 3. The $\mathrm{H}_{2} \mathrm{O}$ abundance in the outer envelope is found to be in the range $0.7-20 \times 10^{-7}$ at $3 \sigma$, the best-fit solution being $2.5 \times 10^{-7}$ (reduced $\chi^{2}=1.3$ ). The inner $\mathrm{H}_{2} \mathrm{O}$ abundance is even more poorly constrained, lower than about $2 \times 10^{-6}$ at $3 \sigma$ 


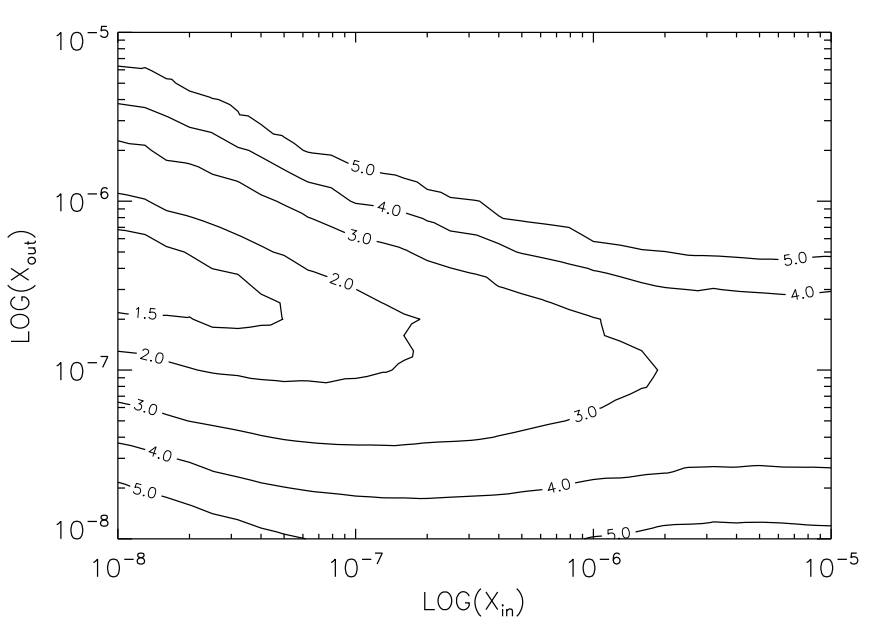

Fig. 5. Reduced $-\chi^{2}$ contours obtained comparing the model predictions and the observations towards IRAS16293, as function of the inner $X_{\text {in }}$ and outer $\mathrm{X}_{\text {out }} \mathrm{H}_{2} \mathrm{O}$ abundance. In these computations, we used the dust temperature and density profiles of the Shu-like model of Table 3.

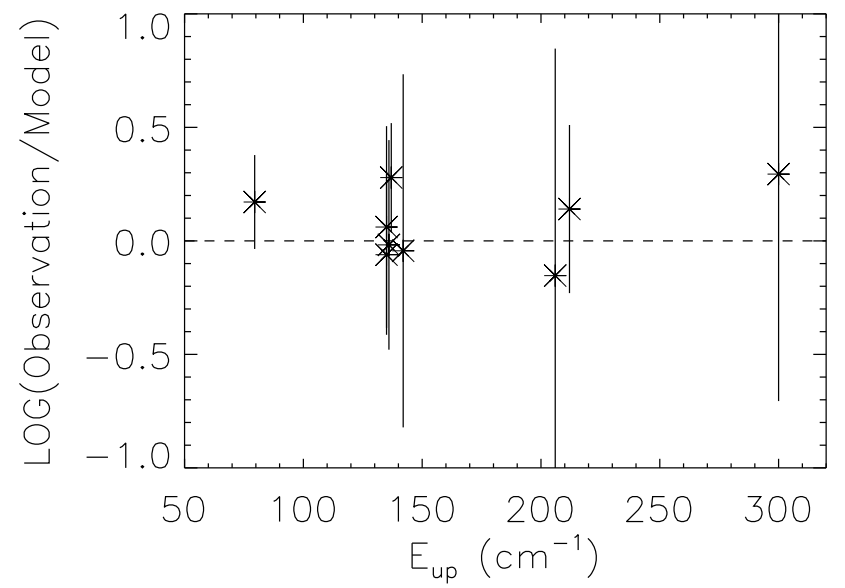

Fig. 6. Ratio of the observed to predicted $\mathrm{H}_{2} \mathrm{O}$ line flux versus the upper level energy in $\mathrm{cm}^{-1}$. In these computations, we used the dust temperature and density profiles of the Shu-like model of Table 3 and an $\mathrm{H}_{2} \mathrm{O}$ abundance equal to $5 \times 10^{-8}$ in the inner envelope and $2.5 \times 10^{-7}$ in the outer envelope (see text).

confidence level, and $0.5 \times 10^{-7}$ at $1.5 \sigma$ confidence level, i.e., slightly lower than the inner abundance, which seems to contradict the hypothesis that ice mantles sublimate injecting water in the gas phase. However, one has to consider that the inner $\mathrm{H}_{2} \mathrm{O}$ abundance is very poorly constrained by the ISO observed lines, which are very optically thick and not at high enough energy (as shown by the predictions of Table 7). We, therefore, only consider significant the $3 \sigma$ level limit to the water inner abundance, cautioning that even that may be questionable for the same reasons (see also Sect. 4). The ratio of the observed by ISO to the predicted lines fluxes as a function of the upper level energy is shown in Fig. 6.

The results for the single power-law density profile are similar, the upper limit to the inner $\mathrm{H}_{2} \mathrm{O}$ abundance being a factor ten lower (because of the higher density in the inner part predicted by this model).

Figure 7 shows the dust and gas temperature profiles for the Shu-like density distribution. Gas and dust are thermally coupled across the whole envelope, the largest difference $(<10 \%)$ between them occuring in the infall region, where $r<r_{\text {inf }}$.
Figure 8 shows the heating and cooling terms across the envelope. The heating is totally dominated by the compression in the entire collapsing region, and by the collisions between the dust and the gas in the outer envelope. We note that the $\mathrm{H}_{2} \mathrm{O}$ and $\mathrm{CO}$ photo-pumping plays only a minor role. The cooling is dominated by the water line emission in the inner region (where the ice sublimate), by the dust-gas collisions in a large intermediate region, and by the $\mathrm{CO}$ line emission in the very outer envelope. These results are very similar to those of Ceccarelli et al. (2000a). We note that low-lying water lines could possibly be contaminated by the outflow driven by the source. This effect could possibly lead to an overestimate of the outer water abundance. Since the ISO water lines are spectrally unresolved, it is impossible to address this question using the ISO data.

Tables 7 and 6 list the predicted fluxes of the water lines that will be observable with $\mathrm{HSO}$, for the best-fit model $\left(\mathrm{H}_{2} \mathrm{O}\right.$ abundance in the outer envelope equal to $2.5 \times 10^{-7}$ ) with $\mathrm{H}_{2} \mathrm{O}$ abundance in the inner envelope equal to $0.5 \times 10^{-7}$, but also for the case with a larger $\mathrm{H}_{2} \mathrm{O}$ inner envelope abundance $\left(2 \times 10^{-6}\right)$.

\section{Discussion and conclusions}

Our new analysis of the single dish and interferometric continuum observations of the envelope of IRAS16293 has confirmed that an envelope of about $2 M_{\odot}$ surrounds the proto-binary system of IRAS16293. The envelope can be described with a Shulike density distribution, corresponding to the gas collapsing towards a $2 M_{\odot}$ central star. The luminosity of IRAS16293 has been re-evaluated to be $22 L_{\odot}$ for a distance of $120 \mathrm{pc}$.

Both the single dish and interferometric data have been able to be reproduced by an envelope with an inner radius between 20 and $30 \mathrm{AU}$, equivalent to about $0.4^{\prime \prime}$ and smaller than the radius at which the dust temperature reaches $100 \mathrm{~K}$ (the ice mantle sublimation temperature) namely 75 to $85 \mathrm{AU}$. We have found that our analysis can reproduce the full SED, including the Spitzer MIR data, without the necessity of a central cavity of $800 \mathrm{AU}$ radius (Jørgensen et al. 2005). The difference between our models and previous ones (based on the Schöier et al. 2002, initial model) is the higher bolometric luminosity (kept as a free parameter in our models) and the lower optical thickness of the envelope $\left(\tau_{100 \mu \mathrm{m}}=2\right.$, namely twice as smaller as in the Schöier's models). These differences help to ensure that the predicted MIR radiation flux agrees with the observed one. Finally, the interferometric data, being dominated by the two components of the binary system, do not provide significant constraints on the envelope structure, with one exception. They exclude the case of an envelope with a single power-law density profile centered on the mid-way point between the two sources. Based on this assumption, Schöier et al. (2004) suggested the presence of a cavity 800 AU in diameter. Since no data constrains where the center of the envelope is located, we favor the solution with the envelope centered on one of the two sources. In addition, the Shu-like model fits the PDBI data slightly better and is thus our preferred solution.

As already noted by Ceccarelli et al. (2000a), the ISO data do not permit us to constrain the inner $\mathrm{H}_{2} \mathrm{O}$ abundance because the detected lines are optically thick and cover a relatively small range of upper-level energies $\left(\leq 300 \mathrm{~cm}^{-1}\right)$. The outer-envelope water abundance is also relatively poorly constrained. In addition, the relatively low spectral resolution of ISO does not allow us to determine whether some lines are contaminated by the emission from the outflow. The future observations with the HIFI spectrometer aboard the Herschel Space Observatory, launched in May 2009, will certainly constrain more tightly the water 


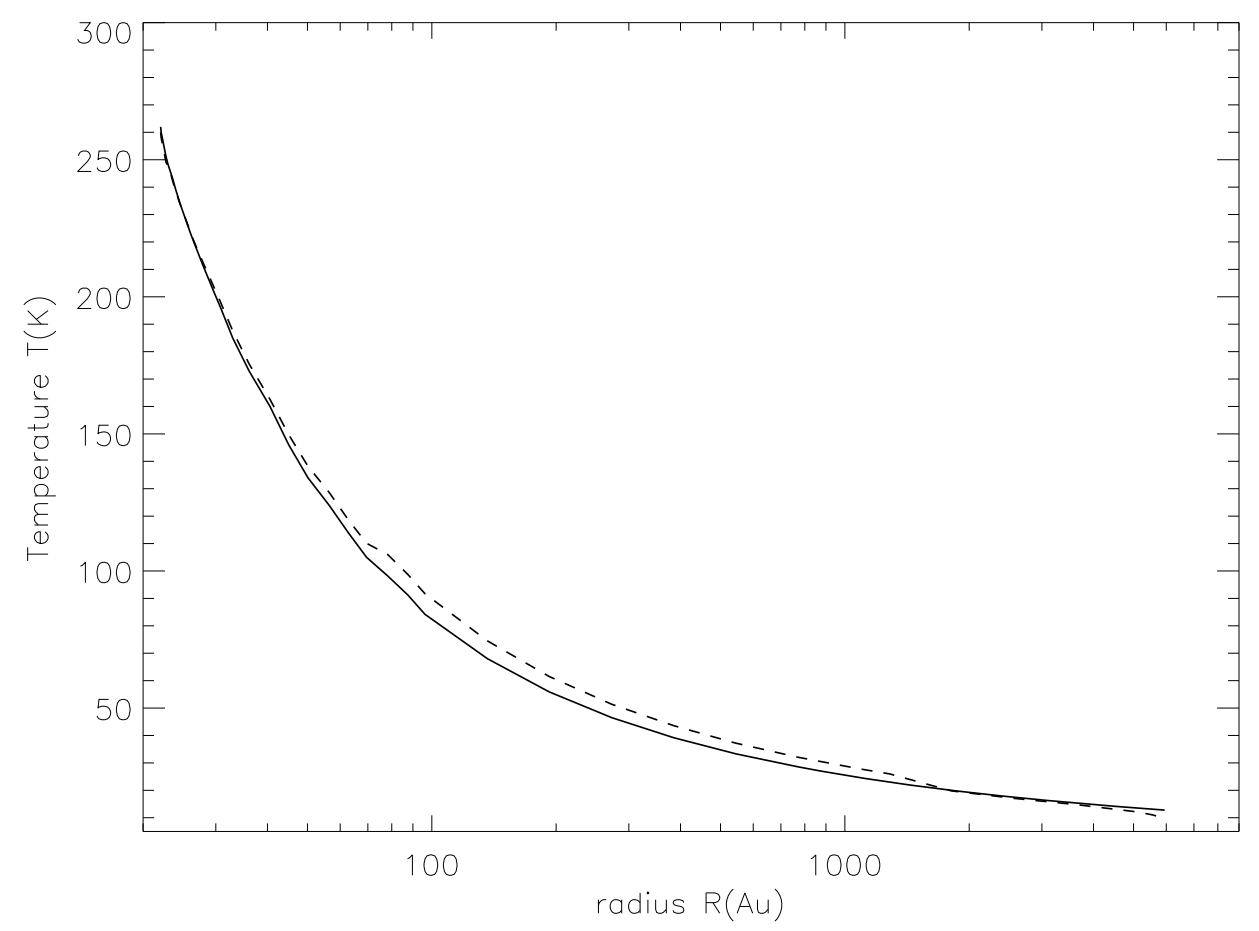

Fig. 7. Dust (solid line) and gas (dashed line) temperature profiles of the best-fit model obtained assuming a Shu-like density distribution (see text).

Table 6. Predictions of the line fluxes (after subtraction of the continuum) of the water lines observable with the Herschel spectrometer HIFI.

\begin{tabular}{|c|c|c|c|}
\hline HIFI range & & $X_{\text {in }}\left(\mathrm{H}_{2} \mathrm{O}\right)=2 \times 10^{-6}$ & $X_{\text {in }}\left(\mathrm{H}_{2} \mathrm{O}\right)=5 \times 10^{-8}$ \\
\hline Transition & $\begin{array}{c}\text { Frequency } \\
(\mathrm{GHz})\end{array}$ & $\begin{array}{c}\text { Flux } \\
\left(\mathrm{K} \mathrm{Km} \mathrm{s}^{-1}\right)\end{array}$ & $\begin{array}{c}\text { Flux } \\
\left(\mathrm{K} \mathrm{Km} \mathrm{s}^{-1}\right)\end{array}$ \\
\hline $1_{10} \rightarrow 1_{01}$ & 557.0 & 32 & 32 \\
\hline $5_{32} \rightarrow 4_{41}$ & 620.7 & 0.31 & 0 \\
\hline $2_{11} \rightarrow 2_{02}$ & 752.0 & 23 & 23 \\
\hline $4_{22} \rightarrow 3_{31}$ & 916.2 & 0.04 & 0.04 \\
\hline $2_{02} \rightarrow 1_{11}$ & 987.9 & 37 & 37 \\
\hline $3_{12} \rightarrow 3_{03}$ & 1097.3 & 26 & 26 \\
\hline $1_{11} \rightarrow 0_{00}$ & 1113.4 & 46 & 46 \\
\hline $7_{25} \rightarrow 8_{18}$ & 1146.6 & 0.04 & 0 \\
\hline $3_{12} \rightarrow 2_{21}$ & 1153.1 & 34 & 34 \\
\hline $6_{34} \rightarrow 5_{41}$ & 1158.3 & 1.0 & 0 \\
\hline $3_{21} \rightarrow 3_{12}$ & 1162.9 & 13 & 11 \\
\hline $4_{22} \rightarrow 4_{13}$ & 1207.6 & 1.1 & 1.1 \\
\hline $2_{20} \rightarrow 2_{11}$ & 1228.8 & 7.6 & 7.6 \\
\hline $5_{23} \rightarrow 5_{14}$ & 1410.7 & 5.7 & 2.1 \\
\hline $6_{43} \rightarrow 7_{16}$ & 1574.2 & 0.10 & 0 \\
\hline $4_{13} \rightarrow 4_{04}$ & 1602.2 & 2.2 & 2.2 \\
\hline $2_{21} \rightarrow 2_{12}$ & 1661.0 & 40 & 37 \\
\hline $2_{12} \rightarrow 1_{01}$ & 1669.9 & 66 & 67 \\
\hline $4_{32} \rightarrow 5_{05}$ & 1713.9 & 2.1 & 0.1 \\
\hline $3_{03} \rightarrow 2_{12}$ & 1716.8 & 56 & 56 \\
\hline $7_{34} \rightarrow 7_{25}$ & 1797.2 & 2.2 & 0.1 \\
\hline $5_{32} \rightarrow 5_{23}$ & 1867.7 & 4.6 & 0.9 \\
\hline $6_{34} \rightarrow 7_{07}$ & 1880.8 & 0.41 & 0 \\
\hline $8_{45} \rightarrow 7_{52}$ & 1884.9 & 0.09 & 0 \\
\hline $3_{31} \rightarrow 4_{04}$ & 1893.7 & 0.02 & 0.02 \\
\hline
\end{tabular}

Notes. The third (fourth) column reports the predictions computed assuming that the inner $\mathrm{H}_{2} \mathrm{O}$ abundance is equal to $2 \times 10^{-6}\left(5 \times 10^{-8}\right)$ and the outer $\mathrm{H}_{2} \mathrm{O}$ abundance is $2.5 \times 10^{-7}$, for the Shu-like model best-fit. 
N. Crimier et al.: The structure of the IRAS16293-2422 envelope

Table 7. Predictions of the line fluxes (after subtraction of the continuum) of the water lines observable with the Herschel spectrometer PACS.

\begin{tabular}{|c|c|c|c|c|}
\hline PACS range & & $X_{\text {in }}\left(\mathrm{H}_{2} \mathrm{O}\right)=2 \times 10^{-6}$ & $X_{\text {in }}\left(\mathrm{H}_{2} \mathrm{O}\right)=5 \times 10^{-8}$ & \\
\hline Transition & $\begin{array}{l}\text { Wavelength } \\
(\mu \mathrm{m})\end{array}$ & $\begin{array}{c}\text { Flux } \\
\left(10^{-12} \mathrm{erg} \mathrm{s}^{-1} \mathrm{~cm}^{-2}\right)\end{array}$ & $\begin{array}{c}\text { Flux } \\
\left(10^{-12} \mathrm{erg} \mathrm{s}^{-1} \mathrm{~cm}^{-2}\right)\end{array}$ & $\begin{array}{c}\text { Flux } \\
\left(10^{-12} \mathrm{erg} \mathrm{s}^{-1} \mathrm{~cm}^{-2}\right)\end{array}$ \\
\hline $9_{36} \rightarrow 8_{45}$ & 62.42 & 0.05 & 0 & \\
\hline $9_{18} \rightarrow 9_{09}$ & 62.93 & 0.4 & 0 & \\
\hline $8_{18} \rightarrow 7_{07}$ & 63.32 & 1.0 & 0.1 & \\
\hline $6_{61} \rightarrow 6_{52}$ & 63.91 & 0.2 & 0 & \\
\hline $7_{61} \rightarrow 7_{52}$ & 63.96 & 0.05 & 0 & \\
\hline $6_{25} \rightarrow 5_{14}$ & 65.17 & 1.7 & 0.5 & \\
\hline $7_{16} \rightarrow 6_{25}$ & 66.09 & 1.2 & 0.2 & \\
\hline $3_{30} \rightarrow 2_{21}$ & 66.44 & 2.5 & 1.6 & \\
\hline $3_{31} \rightarrow 2_{20}$ & 67.09 & 0.3 & 0.3 & \\
\hline $3_{30} \rightarrow 3_{03}$ & 67.27 & 2.0 & 0.5 & \\
\hline $8_{27} \rightarrow 8_{18}$ & 70.70 & 0.6 & 0 & \\
\hline $5_{24} \rightarrow 4_{13}$ & 71.07 & 0.03 & 0.03 & \\
\hline $7_{07} \rightarrow 6_{16}$ & 71.95 & 1.1 & 0.2 & \\
\hline $7_{25} \rightarrow 6_{34}$ & 74.95 & 0.6 & 0 & \\
\hline $3_{21} \rightarrow 2_{12}$ & 75.38 & 2.9 & 2.4 & $2.7 \pm 1.0$ \\
\hline $6_{52} \rightarrow 6_{43}$ & 75.83 & 0.3 & 0 & \\
\hline $5_{50} \rightarrow 5_{41}$ & 75.91 & 0.5 & 0 & \\
\hline $7_{52} \rightarrow 7_{43}$ & 77.76 & 0.2 & 0 & \\
\hline $4_{23} \rightarrow 3_{12}$ & 78.74 & 2.0 & 1.5 & \\
\hline $9_{27} \rightarrow 9_{18}$ & 81.41 & 0.10 & 0 & \\
\hline $6_{16} \rightarrow 5_{05}$ & 82.03 & 1.1 & 0.5 & \\
\hline $8_{36} \rightarrow 8_{27}$ & 82.98 & 0.2 & 0 & \\
\hline $6_{06} \rightarrow 5_{15}$ & 83.28 & 0.04 & 0.04 & \\
\hline $7_{16} \rightarrow 7_{07}$ & 84.77 & 0.8 & 0.1 & \\
\hline $8_{45} \rightarrow 8_{36}$ & 85.77 & 0.06 & 0 & \\
\hline $3_{22} \rightarrow 2_{11}$ & 89.99 & 0.5 & 0.5 & $0.5 \pm 0.5$ \\
\hline $6_{43} \rightarrow 6_{34}$ & 92.81 & 0.3 & 0 & \\
\hline $6_{25} \rightarrow 6_{16}$ & 94.64 & 0.7 & 0.2 & \\
\hline $4_{41} \rightarrow 4_{32}$ & 94.71 & 0.6 & 0.1 & \\
\hline $5_{15} \rightarrow 4_{04}$ & 95.63 & 0.3 & 0.3 & \\
\hline $5_{41} \rightarrow 5_{32}$ & 98.49 & 0.5 & 0 & \\
\hline $5_{05} \rightarrow 4_{14}$ & 99.49 & 1.3 & 1.1 & \\
\hline $5_{14} \rightarrow 4_{23}$ & 100.91 & 1.1 & 0.8 & $1.3 \pm 0.6$ \\
\hline $2_{20} \rightarrow 1_{11}$ & 100.98 & 0.8 & 0.8 & \\
\hline $6_{34} \rightarrow 6_{25}$ & 104.09 & 0.3 & 0 & \\
\hline $2_{21} \rightarrow 1_{10}$ & 108.07 & 2.2 & 2.1 & $1.7 \pm 0.6$ \\
\hline $7_{43} \rightarrow 7_{34}$ & 112.51 & 0.07 & 0 & \\
\hline $4_{14} \rightarrow 3_{03}$ & 113.54 & 1.5 & 1.3 & \\
\hline $7_{34} \rightarrow 6_{43}$ & 116.78 & 0.10 & 0 & \\
\hline $4_{32} \rightarrow 4_{23}$ & 121.72 & 0.4 & 0.2 & \\
\hline $4_{04} \rightarrow 3_{13}$ & 125.36 & 0.6 & 0.6 & \\
\hline $3_{31} \rightarrow 3_{22}$ & 126.71 & 0.1 & 0.1 & \\
\hline $7_{25} \rightarrow 7_{16}$ & 127.88 & 0.10 & 0 & \\
\hline $4_{23} \rightarrow 4_{14}$ & 132.41 & 0.7 & 0.6 & $0.7 \pm 0.7$ \\
\hline $8_{36} \rightarrow 7_{43}$ & 133.55 & 0.03 & 0 & \\
\hline $5_{14} \rightarrow 5_{05}$ & 134.94 & 0.5 & 0.3 & \\
\hline $3_{30} \rightarrow 3_{21}$ & 136.49 & 0.6 & 0.4 & \\
\hline $3_{13} \rightarrow 2_{02}$ & 138.53 & 0.9 & 0.9 & $0.9 \pm 0.7$ \\
\hline $4_{13} \rightarrow 3_{22}$ & 144.52 & 0.1 & 0.1 & \\
\hline $3_{22} \rightarrow 3_{13}$ & 156.20 & 0.2 & 0.2 & \\
\hline $5_{23} \rightarrow 4_{32}$ & 156.26 & 0.3 & 0.04 & \\
\hline $5_{32} \rightarrow 5_{23}$ & 160.51 & 0.1 & 0 & \\
\hline $7_{34} \rightarrow 7_{25}$ & 166.81 & 0.05 & 0 & \\
\hline $3_{03} \rightarrow 2_{12}$ & 174.62 & 1.2 & 1.2 & $2.5 \pm 0.6$ \\
\hline $4_{32} \rightarrow 5_{05}$ & 174.92 & 0.05 & 0 & \\
\hline $2_{12} \rightarrow 1_{01}$ & 179.53 & 1.4 & 1.4 & $2.9 \pm 0.6$ \\
\hline $2_{21} \rightarrow 2_{12}$ & 180.49 & 0.9 & 0.8 & $0.9 \pm 0.4$ \\
\hline $4_{13} \rightarrow 4_{04}$ & 187.11 & 0.05 & 0.05 & \\
\hline
\end{tabular}

Notes. The third (fourth) column reports the predictions computed assuming that the inner $\mathrm{H}_{2} \mathrm{O}$ abundanceis equal to $2 \times 10^{-6}\left(5 \times 10^{-8}\right)$ and the outer $\mathrm{H}_{2} \mathrm{O}$ abundance is $2.5 \times 10^{-7}$, for the Shu-like model best-fit. The fifth column reports the observed ISO fluxes. 

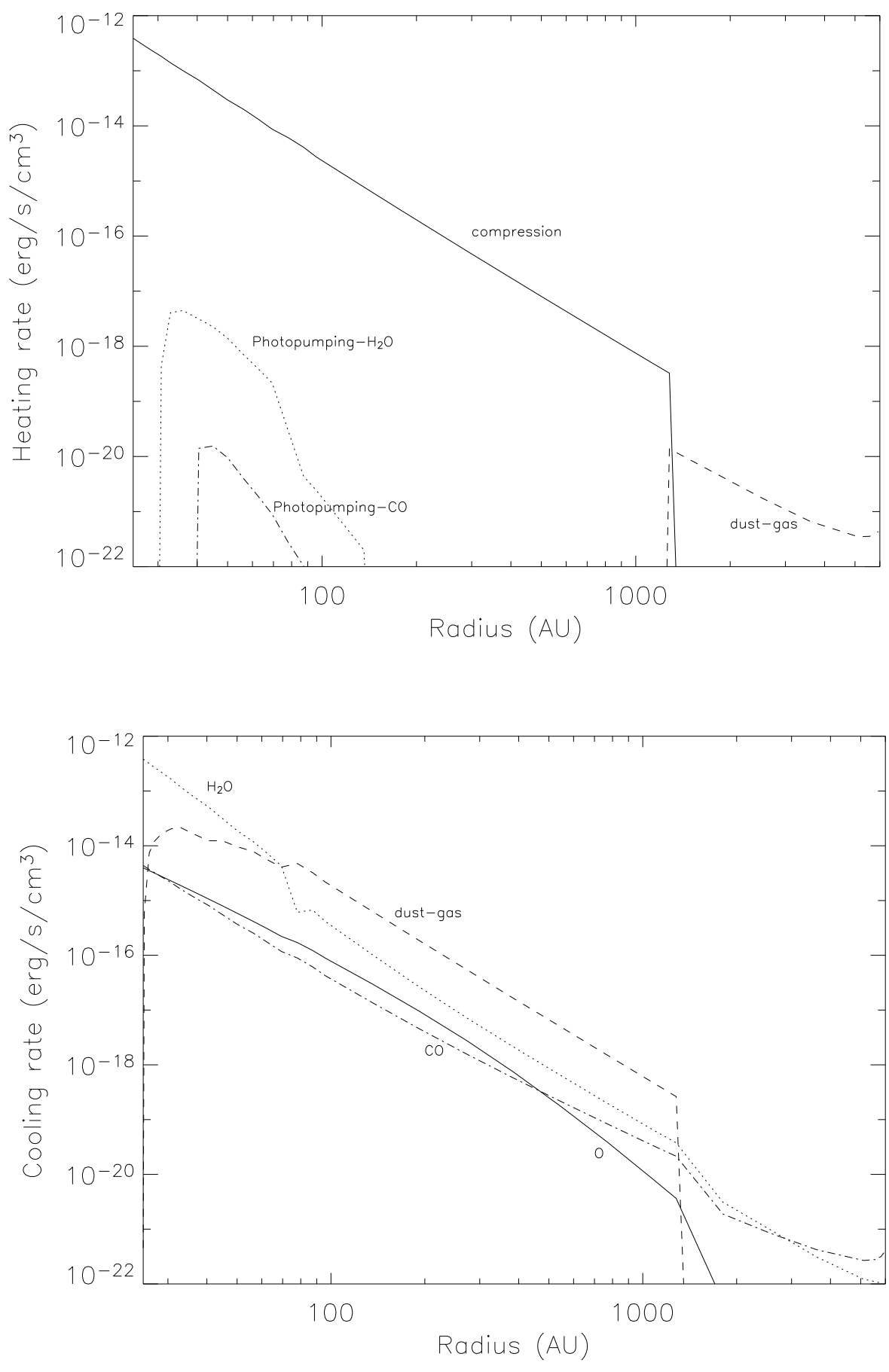

Fig. 8. Heating (top panel) and cooling (bottom panel) rates as function of the radius, computed assuming that the inner $\mathrm{H}_{2} \mathrm{O}$ abundance is equal to $2 \times 10^{-6}$ and the outer $\mathrm{H}_{2} \mathrm{O}$ abundance is $2.5 \times$ $10^{-7}$, for the Shu-like best-fit model. The solid line shows the compression heating (top panel) and the OI line emission (bottom panel). The dust-gas collisions are represented by the dashed curves in both panels. The dotted curves show the contribution of the water molecules and the dotted-dashed curves that of the $\mathrm{CO}$ molecules, in both panels.

abundance profile across the IRAS16293 envelope, helping us to understand the distribution of water in the protostars similar to the Sun's progenitor. On the one hand, the water abundance in the outer envelope $\left(0.7-20 \times 10^{-7}\right)$ derived from the ISO observations here is consistent with some previous estimates of water abundance in cold gas (e.g., Cernicharo et al. 1997) but only marginally consistent with other low estimates (e.g., Snell et al. 2000), so the new Herschel/HIFI observations, with their significantly higher spatial and spectral resolution, will be crucial in settling the question. On the other hand, the inner envelope abundance $\left(<2 \times 10^{-6}\right)$ is lower than expected if all ice in the mantles sublimates (see Ceccarelli et al. 2000a). In this case, the Herschel/HIFI observations will also help us to understand this point.
As stated in the Introduction, the main aim of our study was to provide as accuratly as possible estimates of the dust and gas temperature profiles of the cold envelope of IRAS16293 and its warm inner component, also known as the hot corino, to interpret the data observed in two large projects, TIMASSS and CHESS (see Introduction). We are aware that the proposed description is unable to account for the multiple nature of the IRAS16293 system. However it has the merit of allowing the interpretation of the single-dish observations in the upcoming projects, despite its limitation. A more detailed analysis will only be possible once the relevant molecular emission is observed with interferometers, resolving the two components of the system. Before this, the analysis based on a single warm component and cold envelope is the only viable one and allows a first understanding of 
the chemical composition of a system that will eventually form a star and planetary system like our own.

Acknowledgements. We warmly thank Laurent Loinard for the very useful discussions on the interferometric data of IRAS16293. We acknowledge the financial support by PPF and the Agence Nationale pour la Recherche (ANR), France (contract ANR-08-BLAN-0225). The CSO is supported by the National Science Foundation, award AST-0540882.

\section{References}

Adams, F. C., \& Shu, F. H. 1986, ApJ, 308, 836

Blake, G. A., van Dishoek, E. F., Jansen, D. J., Groesbeck, T. D., \& Mundy, L. G. 1994, ApJ, 428, 680

Bottinelli, S., Ceccarelli, C., Neri, R., et al. 2004, ApJ, 617, L69

Butner, H. M., Charnley, S. B., Ceccarelli, C., et al. 2007, ApJ, 659, L137

Caux, E., Ceccarelli, C., Castets, A., et al. 1999, A\&A, 347, L1

Cazaux, S., Tielens, A. G. G. M., Ceccarelli, C., et al. 2003, ApJ, 593, L51

Ceccarelli, C., Hollenbach, D. J., \& Tielens, A. G. G. M. 1996, ApJ, 471, 400

Ceccarelli, C., Castets, A., Loinard, L., Caux, E., \& Tielens, A. G. G. M. 1998, A\&A, 338, L43

Ceccarelli, C., Castets, A., Caux, E., et al. 2000a, A\&A, 355, 1129

Ceccarelli, C., Loinard, L., Castets, A., Tielens, A. G. G. M., \& Caux, E. 2000b, A\&A, 357, L9

Ceccarelli, C., Maret, S., Tielens, A. G. G. M., Castets, A., \& Caux, E. 2003, A\&A, 410, 587

Ceccarelli, C., Caselli, P., Herbst, E., Tielens, A. G. G. M., \& Caux, E. 2007, in Protostars and Planets V, ed. B. Reipurth, D. Jewitt, \& K. Keil, 47

Cernicharo, J., Lim, T., Cox, P., et al. 1997, A\&A, 323, L25

Chandler, C. J., Brogan, C. L., Shirley, Y. L., \& Loinard, L. 2005, ApJ, 632, 371

Crimier, N., Ceccarelli, C., Lefloch, B., \& Faure, A. 2009, A\&A, 506, 1229

Doty, S. D., \& Neufeld, D. A. 1997, ApJ, 489, 122

Doty, S. D., Schöier, F. L., \& van Dishoeck, E. F. 2004, A\&A, 418, 1021

Dowell, C. D., Allen, C. A., Babu, R. S., et al. 2003, in SPIE Conf. Ser. 4855, ed. T. G. Phillips, \& J. Zmuidzinas, 73

Evans, N. J., \& c2d Team. 2005, BAAS, 37, 1323

Evans, II, N. J., Rawlings, J. M. C., Shirley, Y. L., \& Mundy, L. G. 2001, ApJ, 557,193

Evans, II, N. J., Allen, L. E., Blake, G. A., et al. 2003, PASP, 115, 965
Faure, A., Crimier, N., Ceccarelli, C., et al. 2007, A\&A, 472, 1029 Hunter, T. R. 1997, PhD Thesis, California Institute of Technology Ivezic, Z., \& Elitzur, M. 1997, MNRAS, 287, 799

Jørgensen, J. K., Schöier, F. L., \& van Dishoeck, E. F. 2002, A\&A, 389, 908

Jørgensen, J. K., Lahuis, F., Schöier, F. L., et al. 2005, ApJ, 631, L77

Kovács, A. 2008, in SPIE Conf. Ser., 7020

Lahuis, F., Kessler-Silacci, J. E., Evans, N. J., I., et al. 2006, c2d Spectroscopy Explanatory Supplement, Tech. Rep., Pasadena: Spitzer Science Center

Liseau, R., White, G. J., Larsson, B., et al. 1999, A\&A, 344, 342

Loinard, L., Torres, R. M., Mioduszewski, A. J., \& Rodríguez, L. F. 2008, ApJ, 675, L29

Loinard, L., Rodriguez, L., Pech, G., et al. 2009, BAAS, 41, 296

Maret, S., Ceccarelli, C., Caux, E., Tielens, A. G. G. M., \& Castets, A. 2002, A\&A, 395, 573

Mundy, L. G., Wootten, A., Wilking, B. A., Blake, G. A., \& Sargent, A. I. 1992, ApJ, 385, 306

Ossenkopf, V., \& Henning, T. 1994, A\&A, 291, 943

Parise, B., Simon, T., Caux, E., et al. 2003, A\&A, 410, 897

Parise, B., Ceccarelli, C., \& Maret, S. 2005, A\&A, 441, 171

Sandell, G., \& Weintraub, D. A. 2001, ApJS, 134, 115

Saraceno, P., Andre, P., Ceccarelli, C., Griffin, M., \& Molinari, S. 1996, A\&A, 309,827

Schöier, F. L., Jørgensen, J. K., van Dishoeck, E. F., \& Blake, G. A. 2002, A\&A, 390,1001

Schöier, F. L., Jørgensen, J. K., van Dishoeck, E. F., \& Blake, G. A. 2004, A\&A, 418, 185

Shirley, Y. L., Evans, II, N. J., \& Rawlings, J. M. C. 2002, ApJ, 575, 337

Shu, F. H. 1977, ApJ, 214, 488

Snell, R. L., Howe, J. E., Ashby, M. L. N., et al. 2000, ApJ, 539, L101

Takahashi, T., Silk, J., \& Hollenbach, D. J. 1983, ApJ, 275, 145

Van Der Tak, F. F. S., van Dishoeck, E. F., Evans, II, N. J., Bakker, E. J., \& Blake, G. A. 1999, ApJ, 522, 991

van Dishoeck, E. F., Blake, G. A., Jansen, D. J., \& Groesbeck, T. D. 1995, ApJ, 447,760

Vastel, C., Phillips, T. G., Ceccarelli, C., \& Pearson, J. 2003, ApJ, 593, L97

Walker, C. K., Lada, C. J., Young, E. T., Maloney, P. R., \& Wilking, B. A. 1986, ApJ, 309, L47

Wootten, A. 1989, ApJ, 337, 858

Young, C. H., Shirley, Y. L., Evans, II, N. J., \& Rawlings, J. M. C. 2003, ApJS, 145,111 